\title{
Combined portal vein and hepatic vein embolization - finally the platinum procedure of regenerative liver surgery?
}

\author{
Erik Schadde ${ }^{1,2,3}$ \\ ${ }^{1}$ Institute of Physiology, University of Zurich, Zurich, Switzerland; ${ }^{2}$ Department of Surgery, Division of Transplant Surgery, Rush University Medical \\ Center, Chicago, Illinois, USA; ${ }^{3}$ Department of Surgery, Cantonal Hospital Winterthur, Zurich, Switzerland \\ Correspondence to: Erik Schadde, MD, FACS, FEBS (HPB). Institute of Physiology, University of Zurich, Winterthurerstr. 190, CH-8057 Zurich, \\ Switzerland. Email: erik.schadde@uzh.ch.
}

Submitted Sep 16, 2019. Accepted for publication Sep 25, 2019.

doi: $10.21037 /$ hbsn.2019.09.20

View this article at: http://dx.doi.org/10.21037/hbsn.2019.09.20

Regenerative liver surgery is the field of research and clinical practice which tries to increase the volume of the future liver remnant prior to resection. Too small liver remnants lead to post-hepatectomy liver failure after extensive resections, a syndrome of portal flow congestion, progressive cholestasis, lack of synthetic function, renal failure, ascites, encephalopathy and ultimately death from infection. What is too small, is not entirely clear, depends on many patient, liver and surgery factors, but is generally between $20-40 \%$. The field of regenerative liver surgery expanded considerably over the last years and has become the favorite playground for surgeons who like to push the limits of resectability of liver tumors. Skeptical and weary oncologists at multidisciplinary tumor boards have become used to the sight of liver surgeons who consider no extent of disease off limits, equipped with a growing armada of eponyms like "portal vein embolisation (PVE)", "portal vein ligation (PVL)", "associating liver partition and PVL for staged hepatectomy (ALPPS)", "associating liver tourniquet and PVL for staged hepatectomy (ALTPS)", "partial ALPPS (PALPPS)", "radiofrequency-assisted liver partition and portal vein ligation for staged hepatectomy (RALPPS)" and "Mini-ALPPS". These eponyms project hermetic competence and revive the long-lost optimism of aggressive cancer surgery in the age of genetics and targeted therapies.

The infatuation with this new frontier starts with the discovery of gold by a German group of surgeons, who showed in 2012 that rapid liver growth of $80 \%$ increase within 1 week (similar to the growth seen after partial hepatectomy) can be achieved prior to resection by transecting the liver at the time of portal vein occlusion in the first stage of a two-stage hepatectomy (1). Extended resection followed after only 10 days. This is in stark contrast to portal vein embolization, which generally achieves a $40 \%$ increase in 6 weeks (2).

The Germans proposed the technique as a way out in cases where the surgeon had committed to exploration, but could not technically resect without a high risk of posthepatectomy liver failure. The gold rush that followed, however, was triggered by marketing of the "in-situ splitprocedure", as invented by the Germans, under the new brand "ALPPS". Over the next years ALPPS would be an endless source of arguments the be had, and papers to be written $(3,4)$. The novel operation suggested that a central problem of the surgical oncology of the liver had finally been solved forever. After the currency "ALPPS" had been coined in an editorial by de Santibanes and Clavien in Annals of Surgery (5), a wave of enthusiasm for the novel procedure ensued.

No calls of warning from the large volume centers like MD Anderson (6) and Hospital Beaujon (7) in Paris could dissuade liver surgeons to try out this somewhat experimental procedure in an estimated few thousands of patients under the banner of "innovation" (8). Disillusionment soon set in when the first studies based on a registry clearly showed what the early sceptics had suspected all along. The two-stage hepatectomy ALPPS was not safe for primary liver tumors $[12 \%$ and $27 \%$ mortality for hepatocellular carcinoma (HCC) and primary hepatocellular carcinoma (PHCC) respectively] and the mortality and morbidity rate for colorectal liver metastases was also higher than acceptable by contemporary standards 
(8\%) (9). An early randomized trial in Zürich had to be stopped and restarted (ClinicalTrials.gov Identifier: NCT02758977). Was the procedure too complex or is there an intrinsic problem with rapid hypertrophy induced by in-situ split? Some answers came from studies of ALPPS patients using liver function assessment with hepatobiliary iminodiacetic acid (HIDA) scintigraphy (10). These studies raised the scepter of doubt if volume increase in the liver remnant alone also correlated with functional increase and thereby created quantity, but not quality of liver tissue, a problem new to liver regeneration.

In the meantime, a quiet team of Scandinavian evidence workers dissected the ailing body of ALPPS in a successful prospective randomized study of ALPPS $v s$. PVE, exclusively in patients with colorectal liver metastasis (CRLM) (10). They found that the principles and foundations of the in-situ split procedure were not so bad after all. Per Sandström from Linköping and his coworkers from Stockholm, Gotenburg, Lund, Kopenhagen and Oslo showed in their randomized LIGRO trial of ALPPS vs. PVE in patients with bi-lobar CLRM that surgical resectability indeed increased from $57 \%$ to $92 \%$ when ALPPS was used as the primary strategy. Abrogation of portal vein collaterals between liver lobes, the principle of ALPPS, indeed had a clinical utility. However, even in the hand of high-volume surgeons with conservative indications in the quite centralized Scandinavian system, the 90-day mortality of the procedure remained high, at $8.3 \%$ (10). The question remained, why a procedure that was destined to solve the problem of post-hepatectomy liver failure via preoperative volume growth exposed such a high number of patients to post hepatectomy liver failure (11).

Our group drew the conclusion that the procedure was not ready for prime time and we resorted to a large animal preclinical model in the pig. We demonstrated that the disruption of the naturally occurring portal vein collateralization after embolization is the key physiological event resulting in the rapid hypertrophy of ALPPS (12). To disrupt these collaterals, the knife is not needed at all. Portal collateralization and neoangiogenesis are likely the result of portal hyper flow restricted by a limited venous outflow bed in the growing lobe. It can therefore be abrogated by occluding the venous outflow on the side of portal inflow occlusion. In the preclinical pig model, simultaneously performed portal vein and hepatic vein ligation abrogates collaterals almost as efficiently as transection and rapid hypertrophy is as robust as in the ALPPS model in pigs (13).
While considering the practical implications of these findings, we came across a series of studies published from a group of interventional radiologists from the University of Montpellier. Guiu et al. had convincingly demonstrated that combined simultaneous embolization of portal vein and hepatic veins allowed to optimize the hypertrophy effect of PVE (14). It also allowed him to create a new eponym, liver venous deprivation (LVD). The Montpellier technique includes the meticulous occlusion of venous collaterals observed immediately after large vessel occlusion using n-butyl cyanoacrylate. With an intriguing mixture of technical innovation and analytic precision, Guiu et al. showed that their new procedure solved the volumefunction dilemma of ALPPS since functional HIDA scans of the growing liver showed congruence of volume and function (15). The endovascular approach abolished the necessity of two-stage surgery and was highly attractive to reduce the surgical severity of the ALPPS of two major surgical interventions in close sequence.

The new study "Perioperative impact of liver venous deprivation...", published in this issue of $H B S N$, focuses on surgical outcomes and represents the first retrospective attempt at comparing LVD with PVE from the surgical perspective within the Montpellier group. While the case mix is highly heterogenous (CRLM, HCC and "others") and the numbers are too small to avoid a type II error in what is basically a noninferiority claim of surgical outcomes between the procedures, the message is still very clear. The simultaneous endovascular occlusion of portal and hepatic veins may potentially avoid the problems of ALPPS, while preserving the benefit of rapid hypertrophy as evidenced by an almost 4-time increased kinetic growth rate and a high feasibility rate. The study fails to show a shorter time between the procedure and resection because the authors report that they could not reduce the time to surgery in LVD "due to administrative constraints", however, these limitations are clearly stated by the authors. Is the novel "endovascular ALPPS" just another gold coin minted in the ALPPS-factory? Or is it the platinum procedure of regenerative liver surgery envisioned by those of us who believe regenerative liver surgery indeed changes the limitations of resectability? The preliminary reports like this one suggest that this needs to be explored further in collaborative randomized trials. Two such trials are underway, one in France and one international with more than 30 centers, the DRAGON trial based in Maastricht, Netherlands, which 
also includes the Scandinavians collaborating in LIGRO. Hopefully we will get more evidence about regenerative liver surgery soon.

\section{Acknowledgments}

None.

\section{Footnote}

Conflicts of Interest: The author has no conflicts of interest to declare.

Ethical Statement: The author is accountable for all aspects of the work in ensuring that questions related to the accuracy or integrity of any part of the work are appropriately investigated and resolved.

\section{References}

1. Schnitzbauer AA, Lang SA, Goessmann H, et al. Right portal vein ligation combined with in situ splitting induces rapid left lateral liver lobe hypertrophy enabling 2-staged extended right hepatic resection in small-for-size settings. Ann Surg 2012;255:405-14.

2. van Lienden KP, van den Esschert JW, de Graaf W, et al. Portal vein embolization before liver resection: a systematic review. Cardiovasc Intervent Radiol 2013;36:25-34.

3. Olthof PB, Schadde E. The rush to novelty and high expectations in surgery: the case of ALPPS. J Clin Transl Res 2016;2:79-83.

4. Olthof PB, Schnitzbauer AA, Schadde E. The HPB controversy of the decade: 2007-2017 - Ten years of ALPPS. Eur J Surg Oncol 2018;44:1624-7.

5. de Santibanes E, Clavien PA. Playing Play-Doh to prevent postoperative liver failure: the "ALPPS" approach. Ann

Cite this article as: Schadde E. Combined portal vein and hepatic vein embolization-finally the platinum procedure of regenerative liver surgery? Hepatobiliary Surg Nutr 2020;9(1):92-94. doi: 10.21037/hbsn.2019.09.20
Surg 2012;255:415-7.

6. Aloia TA, Vauthey JN. Associating liver partition and portal vein ligation for staged hepatectomy (ALPPS): what is gained and what is lost? Ann Surg 2012;256:e9; author reply e16-9.

7. Dokmak S, Belghiti J. Which limits to the "ALPPS" approach? Ann Surg 2012;256:e6; author reply e16-7.

8. Belghiti J, Dokmak S, Schadde E. ALPPS: Innovation for innovation's sake. Surgery 2016;159:1287-8.

9. Schadde E, Ardiles V, Robles-Campos R, et al. Early survival and safety of ALPPS: first report of the International ALPPS Registry. Ann Surg 2014;260:829-36; discussion 836-8.

10. Olthof PB, Tomassini F, Huespe PE, et al. Hepatobiliary scintigraphy to evaluate liver function in associating liver partition and portal vein ligation for staged hepatectomy: Liver volume overestimates liver function. Surgery 2017;162:775-83.

11. Schadde E, Raptis DA, Schnitzbauer AA, et al. Prediction of Mortality After ALPPS Stage-1: An Analysis of 320 Patients From the International ALPPS Registry. Ann Surg 2015;262:780-5; discussion 785-6.

12. Deal R, Frederiks C, Williams L, et al. Rapid Liver Hypertrophy After Portal Vein Occlusion Correlates with the Degree of Collateralization Between Lobes-a Study in Pigs. J Gastrointest Surg 2018;22:203-13.

13. Schadde E, Guiu B, Deal R, et al. Simultaneous hepatic and portal vein ligation induces rapid liver hypertrophy: A study in pigs. Surgery 2019;165:525-33.

14. Guiu B, Chevallier P, Denys A, et al. Simultaneous transhepatic portal and hepatic vein embolization before major hepatectomy: the liver venous deprivation technique. Eur Radiol 2016;26:4259-67.

15. Guiu B, Quenet F, Escal L, et al. Extended liver venous deprivation before major hepatectomy induces marked and very rapid increase in future liver remnant function. Eur Radiol 2017;27:3343-52. 